\title{
A Case of Postoperative Covid-19 Infection After Cardiac Surgery: Lessons Learned
}

\author{
Giuseppe Rescigno, MD, FETCS, ${ }^{1}$ Michael Firstenberg, $M D,{ }^{2}$ Igor Rudez, $\mathrm{MD}, \mathrm{PhD},{ }^{3}$ \\ Mauin Uddin, MD,${ }^{1}$ Kumar Nagarajan, $M D,{ }^{1}$ Nicholas Nikolaidis, MD, FRCS ${ }^{1}$ \\ ${ }^{1}$ Cardiothoracic Surgery Department, New Cross Hospital, Wolverhampton, United Kingdom; ${ }^{2}$ Department of \\ Cardiothoracic and Vascular Surgery, The Medical Center of Aurora, Aurora, CO; ${ }^{3}$ Department of Cardiac and Transplant \\ Surgery, Dubrava University Hospital, Zagreb, Croatia
}

\section{ABSTRACT}

While the focus of the medical community is on the management of COVID-19 and its associated complex presentations, it is critical to recognize that patients will continue to present with other medical problems that require urgent therapeutic interventions. There is growing concern that such interventions might have an impact on the natural history of COVID-19. We present a case of a patient who presented with unstable angina and multivessel coronary artery disease for which coronary artery bypass surgery was indicated and performed. Unfortunately, he succumbed to respiratory complications attributed to COVID-19. Our experience suggests concern about adverse outcomes in patients undergoing cardiac surgery who might be infected with COVID-19. Clearly, additional investigations and experience are needed.

\section{INTRODUCTION}

On December 31, 2019, the Chinese Health Authority warned the World Health Organization (WHO) about a series of unusual pneumonia cases in the city of Wuhan. At the time, the etiology was unknown. On January 5, 2019, Chinese officials ruled out the possibility that this was a recurrence of the severe acute respiratory syndrome (SARS) virus. Two days later, a novel virus from the coronavirus family was identified as the likely agent and was termed 2019-nCoV. Since then, the infection, called COVID-19 (coronavirus disease 2019), has evolved into a global pandemic of massive proportions, with $>120,000$ deaths as of April 14, 2020.

In the United Kingdom, the first cases were recorded at the end of January [Ball 2020]. At that time, there was uncertainty about the best response to a possible outbreak. Whereas many countries had adopted a policy of selfdistancing and lockdown, some countries opted for alternative

Received April 13, 2020; accepted April 13, 2020.

Correspondence: Giuseppe Rescigno, Consultant Cardiac Surgeon, Cardiothoracic Surgery Department, New Cross Hospital, Wolverhampton Road, Wolverbampton WV10 0QP, UK (e-mail: giuseppe.rescigno@nbs.net). approaches to management [gov.uk 2020]. Unfortunately, the National Health Service was rapidly overwhelmed by patients affected by COVID-19, as were many other health organizations of the world. Hospitals faced a significant reduction of intensive care beds; with concerns of resource availability, combined with attempts to minimize nosocomial spread, many countries implemented policies that limited non-urgent/emergent procedures.

Although surgical volumes have dropped substantially, patients with potentially life-threatening problems, such as cancers and unstable cardiovascular disease, still need guideline-directed timely and appropriate management. However, the potential impact of concomitant COVID-19 infection on patients who undergo urgent or emergency procedureseven when risk stratified with existing scoring systems-is poorly defined. This case report describes a routine cardiac surgery operation with a poor outcome due to postoperative COVID-19, and the changes in our practice during these difficult times.

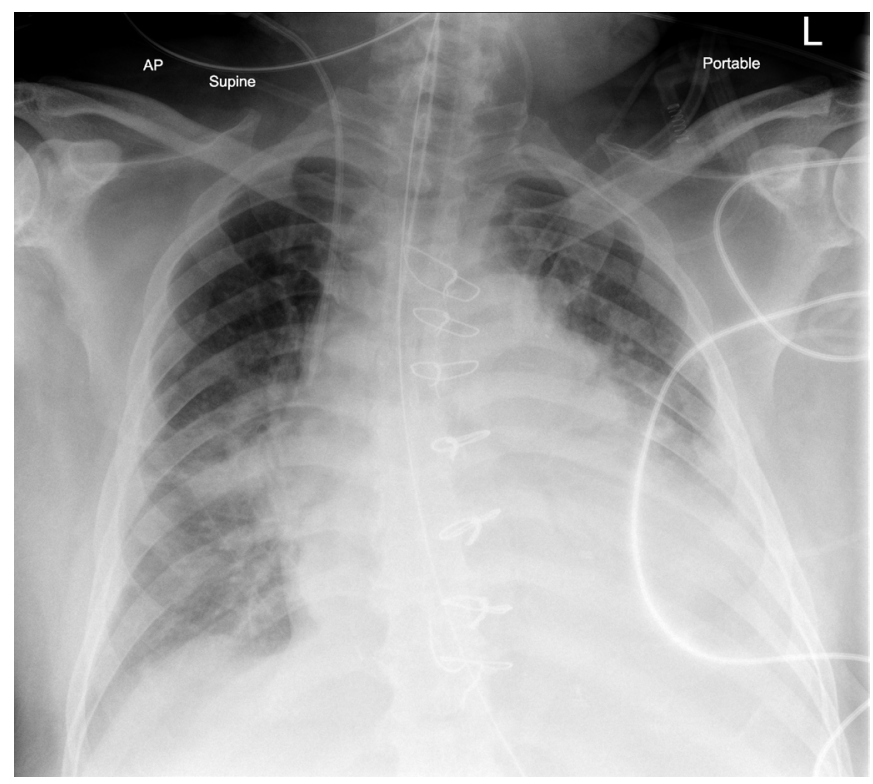

Chest radiogram shows severe bilateral lung involvement from the infective process. 
Arterial Blood Gases and Modality of Ventilation*

\begin{tabular}{|c|c|c|c|c|c|c|c|c|c|c|}
\hline Mode & Face mask & BiPAP & BiPAP & BIPAP & BIPAP & SIMV & SIMV & SIMV & SIMV & SIMV \\
\hline $\mathrm{pH}$ & 7.33 & 7.44 & 7.42 & 7.39 & 7.36 & 7.29 & 7.39 & 7.34 & 7.356 & 7.32 \\
\hline $\mathrm{PaCO}_{2}(\mathrm{kPa})$ & 5.8 & 4.64 & 5.2 & 4.98 & 5.80 & 7.16 & 5.96 & 6.71 & 6.52 & 6.09 \\
\hline Base excess (mEq/L) & -3.3 & -0.5 & 0.3 & -4.3 & -1.4 & -1.8 & 1.8 & 0.8 & 0.7 & -3.1 \\
\hline Lactate (mmol/L) & 1.9 & 1.4 & 1.6 & 0.93 & 1.08 & 1.6 & 1.41 & 1.09 & 1.26 & 1.43 \\
\hline $\mathrm{HCO}_{3}(\mathrm{mmol} / \mathrm{L})$ & 21.7 & 23.9 & 24.7 & 20.6 & 24.1 & 25.4 & 25.9 & 25.0 & 24.9 & 23.1 \\
\hline $\mathrm{SaO}_{2}(\%)$ & 96 & 92 & 93 & 93.7 & 89.9 & 87.1 & 86.5 & 86.5 & 86.9 & 86.4 \\
\hline Hemoglobin (g/L) & 149 & 149 & 148 & 119 & 136 & 121 & 119 & 120 & 127 & 128 \\
\hline
\end{tabular}

*BiPAP indicates bilevel positive airway pressure; $\mathrm{FiO}_{2}$, fraction of inspired oxygen; $\mathrm{HCO}_{3}$, bicarbonate; $\mathrm{PaCO}_{2} / \mathrm{O}_{2}$ partial pressure of carbon dioxide/oxygen; $\mathrm{SaO}_{2}$, oxygen saturation; $\mathrm{SIMV}$, synchronized intermittent mandatory ventilation.

\section{CASE REPORT}

Our patient was a 63 -year-old white male who was admitted to a secondary referring hospital of our region with unstable angina. Troponin levels were within the normal range, and a coronary angiogram showed severe triple vessel disease. He had mild left ventricular dysfunction, and cardiovascular risk factors included hypertension, type 2 diabetes mellitus, family history, and morbid obesity (body mass index 39.3). He had a history of sleep apnea, excessive alcohol consumption, and previous stroke without sequelae. Computed tomography of the chest showed mild signs of chronic obstructive pulmonary disease only. Based on National Health Service recommendations at the time of admission, preoperative COVID19 assessment was not performed, as he had no recent fevers, his inflammatory markers were normal (C-reactive protein 3 $\mathrm{mg} / \mathrm{L}$ ), and he did not complain of any symptoms suggestive of a respiratory infection. The multidisciplinary team discussion reached a consensus for surgical revascularization.

Ten days later, after medical optimization and stabilization, the patient was transferred to our institution for surgery. Because of a hospital shortage of intensive care unit (ICU)/ intensive treatment unit (ITU) beds due to the pandemic, his surgery was delayed another 5 days. His interval hospital course was uneventful, and he was subsequently taken for coronary artery bypass surgery.

After uneventful induction of general anesthesia, intraoperative transesophageal echocardiogram showed a moderately impaired right ventricle with moderate to severe tricuspid regurgitation that was only mild at the transthoracic cardiac echo. He underwent routine aorta-bicaval cannulation for cardiopulmonary bypass, and myocardial protection with cold blood antegrade cardioplegia was used. Coronary revascularization consisted of the left internal mammary artery to the left anterior descending artery and reverse segment saphenous veins grafts to an obtuse marginal circumflex and posterior descending arteries. A tricuspid annuloplasty was performed with a $28-\mathrm{mm}$ Tri-Ad ring (Medtronic, Minneapolis, MI). The patient was weaned from cardiopulmonary bypass without difficulty, and repeat echocardiography showed no residual tricuspid regurgitation. Heparin was reversed with protamine sulfate, and the patient was transferred to the ICU, where he was extubated without difficulty 3 hours later. No blood products were used perioperatively.

The patient's initial postoperative course was uneventful. On postoperative day 1, however, he experienced severe hypoxia and dyspnea with fever. After a failed attempt to improve his condition by noninvasive ventilation support, the patient required emergency reintubation. Bronchialalveolar lavage was positive for SARS2-CoV2 RNA. Chest $\mathrm{x}$-ray showed bilateral consolidation (Figure).

A complete infective screening test (urine, blood, and sputum culture) was carried out without any positive result. Cardiac echocardiogram did show good left ventricular function and moderately impaired right ventricular function. Arterial blood gas results and $\mathrm{O}_{2}$ requirements are shown in the Table. His ventilatory status continued to deteriorate, and he died on postoperative day 9 . The presumed cause of death was severe postoperative COVID-19 pneumonia.

\section{DISCUSSION}

The COVID-19 pandemic is globally overwhelming health care and economic resources. Cardiac surgery has been particularly affected by the virus outbreak, as intensive care and hospital critical care resources are limited, which has prompted elective procedures to be delayed and consideration given toward lesser therapies. Even patients who require urgent or emergent procedures face challenges from a growing scarcity of available resources. 
Moreover, the ideal approach for pandemic management is lacking, and clinical, administrative, and political decisionmaking has been subject to intense criticism [Horton 2020]. In the early phases, indications and availability for testingand even concerns of a large percentage of false negativespotentially contributed to the rapid disease progression.

This patient was, unfortunately, a victim of our initial lack of experience in management of this disease. The patient was not tested preoperatively, and hence we were not aware of his COVID-19 status. The waiting time in hospital was clearly too long: the patient was referred on March 4, and the operation was done 15 days later, all delays that potentially could have exposed him to a nosocomial infection. Had our team been aware of his infection and the inexperience with the cardiac surgical implications of this growing comorbidity, alternative therapies, such as high-risk percutaneous coronary intervention, would have been advocated.

In this regard, we now have a well-defined policy for cardiac surgery procedures during the pandemic that is summarized in the following points:

- Elective cases have been temporarily suspended.

- Two ITU beds on our ward protect patients from cross-infection.

- Urgent cases include a preoperative assessment of COVID-19 status (nasal and throat swabs). If the patient is negative, the operation can be performed with reasonable safety. In addition, postintubated lavage is performed after intubation to detect any false-negative tests.

- Urgent cases are not delayed because of preoperative antiplatelet use. We believe the risk-benefit analysis of an increased risk for bleeding and need for transfusion (acknowledging that blood and products are becoming a limited resource during this pandemic as well) versus a possible COVID-19 infection favors early surgery.

- We have adopted a more aggressive approach in terms of early extubation (including potentially in the operating room) and earlier hospital discharge to maximize resources.

- Emergency surgery is performed regardless of the above, unless there is symptomatic preoperative COVID-19 status. In such cases, alternative therapies are considered, if possible.

Our local policies mirror in part what has been recently suggested by the Society of Cardiothoracic Surgery of Great Britain and Ireland (SCTS) [SCTS 2020a].
The comorbidities and postoperative status of our patient were complex, and the cause of his death, although attributed to COVID-19, could have been multifactorial. Our case does raise the growing concern that an undiagnosed or potentially concomitant COVID-19 infection might precipitate a catastrophic pathologic response refractory to standard treatments after cardiac surgery. As such, our case illustrates the growing challenges clinicians-and especially cardiothoracic surgeons-face in this era, in which concomitant viral infection may yield potentially unpredictable physiologic responses.

In response, the Society for Thoracic Surgeons (STS) has included additional database fields specifically addressing potential COVID-19 infection, and COVID-19-positive patients are currently excluded from STS publicly reported outcomes [SCTS 2020b]. Clearly, more data and experience with the cardiac surgical implications of COVID-19 are necessary for critical decision-making. Current guidelines, protocols, risk scoring systems, and clinical practice might require substantial modifications to account for potential COVID-19 infections.

\section{REFERENCES}

Ball T, Wace C. January 31, 2020. Hunt for contacts of coronavirusstricken pair in York. The Times. Available at: https://www.thetimes. co.uk/article/hunt-for-contacts-of-coronavirus-stricken-pair-in-yorkdh363qf8k. Archived from the original on 4 February 2020. Retrieved 31 March 2020.

Coronavirus (COVID-19): latest information and advice. 2020. GOV. UK. Available at: https://www.gov.uk/guidance/coronavirus-covid19-information-for-the-public. Archived from the original on 8 March 2020. Retrieved 31 March 2020.

Horton R. 2020. Offline: COVID-19 and the NHS-"a national scandal." Lancet 2020;395:1022.

Society of Cardiothoracic Surgery of Great Britain and Ireland (SCTS). March 23, 2020. Guidance document to UK cardiac and thoracic teams for procedures on patients with COVID-19. Available at: https://scts. org/wp-content/uploads/2020/03/SCTS-ACTACC-SCPS-TheatreCOVID-pathway-Final.pdf.

Society of Cardiothoracic Surgery of Great Britain and Ireland (SCTS). March 26, 2020. Collection of data for national audits during the COVID-19 emergency and recovery phases. Letter to membership. Available at: https://scts.org/scts-letter-to-the-membership-on-datacollection-for-national-audits-during-the-covid-19-outbreak-26thmarch-2020/. 